\title{
Application of statistical filtering for optical detection of tool wear
}

\author{
M. Sortino * \\ Department of Electrical, Management and Mechanical Engineering, University of Udine, Udine 33100, Italy
}

Received 22 July 2002; received in revised form 14 October 2002; accepted 29 October 2002

\begin{abstract}
The application of automated tool condition monitoring systems is very important for unmanned machining systems. Tool wear monitoring is a key factor for optimization of the cutting processes. Basically, tool wear monitoring systems can be subdivided into two classes: direct and indirect. Currently direct tool wear monitoring systems are most frequently based on machine vision by camera. Several approaches have been studied for tool wear detection by means of tool images, and an innovative statistical filter proved to be very efficient for worn area detection. A new approach has been implemented and tested in order to develop an automatic system for tool wear measurement. This new approach is described in this paper and the main topics related to tool wear monitoring using wear images have been discussed.
\end{abstract}

(C) 2003 Elsevier Science Ltd. All rights reserved.

Keywords: Tool condition monitoring; Tool wear; Computer vision

\section{Introduction}

Today cutting processes play a large role in supporting the economies of developed countries. Machining operations like turning, milling and drilling are involved in the production of most of the goods that are used everyday. This simple statement explains how important is it to study these operations and how much can be spared by choosing cutting parameters close to the optimum values.

Nowadays the machining industry is undergoing several trends. The main trend is toward production cost reduction by using higher cutting speeds and by reducing human resources. The industry trends are toward unmanned machining systems where the importance of an adequate calibration of working parameters is even more important because machines cannot substitute human learning capabilities. Much research effort has been made in developing and applying thermal, force, vision, acoustic and acceleration sensors to machines and by implementing 'intelligent' systems to detect directly or indirectly the machining condition from acquired signals.

\footnotetext{
* Tel.: +39-0432-558251; fax: +39-0432-558251.

E-mail address: sortino@uniud.it (M. Sortino).
}

State of the art of sensing systems and their industrial application have been investigated by the Work Group for Tool Condition Monitoring of CIRP and the results have been presented by Byrne et al. [1]. All the known systems that can be used for this purpose have been described and also their applicability has been discussed. The main point that has been achieved is that most failures are due to inadequate or wrong monitoring system set-up or operator interface misunderstandings, thus underlining the necessity of systems very simple to use, possibly avoiding direct operator interaction. Recently, state of the art of sensors for tool condition monitoring has been discussed by Kuljanic and Sortino [2].

It has been demonstrated that the key factor for production characterization is tool wear, and it must be monitored very carefully. It is also important to understand that the direct measurement of wear requires experienced personnel, its accuracy depends on the personnel capabilities and thus it is not easily repeatable. In the modern machining centre, where human interaction is reduced to a minimum, there is a need for the machine to be able to recognize automatically the wear condition of the tool [3].

Research efforts have been made in developing and applying thermal, force, vision, acoustic and acceleration sensors to machines and by implementing intelligent systems to detect directly or indirectly the tool wear con- 
dition and their application and characteristics have been discussed by Dimla and Dimla [4]. Tool wear estimation systems can be subdivided into two main classes: direct and indirect. Direct tool wear estimation systems are able to measure directly the tool wear by means of tool images, tactile sensors, etc. Their application is very simple and the reliability is high. In the past, several methodologies to determine the wear land were used such as by using a profile projector, an optical section microscope, surface analysers and radioactive sensors, as described in Ref. [5]. Indirect tool wear estimation methods are based on measurement and comparison of signals (for example, cutting forces) that are connected indirectly to tool wear. The main advantage of indirect methods is that they are applied online. Unfortunately, these methods are very complex to design and apply because of the lack of knowledge of how wear can affect the measured signal, the geometry of cutting and the unpredictable behaviour of the wear process itself. Moreover, the application of these methods has a limited reliability and the sensor cost is still very high.

However, the automated application of direct tool wear estimation system is difficult because the detection system should be able to detect the wear zone and measure it. This problem is very complex as the wear zone has noisy contours and a lot of disturbing failures can be present (for example, scraps, lubricant, reflections, etc.). Therefore, the detection software must be carefully designed. In this paper, an innovative algorithm for tool wear zone identification and a detection system is presented.

\section{Direct methods for tool wear estimation}

Direct measurement of tool wear can be made either by means of optical devices or by using a tactile sensor. The application of tactile sensors is not very common, while optical devices such as toolmaker microscopes and cameras are more practical and their cost is low. The basic system for tool wear measurement using optical devices is illustrated in Fig. 1.

A camera with an appropriate lens is used in the field to capture an image of tool wear, which is stored and processed by a computer. The most common algorithm used to detect the worn zone from the image is a highpass bi-dimensional filter, which enhances the edges. Then the worn zone can be identified and measured. Finally, the measurements can be taken according to ISO3685 and ISO8688-1 and 2. The main steps of the worn-zone detection are illustrated in Fig. 2.

During image acquisition the illumination of the tool should be made very carefully to avoid the presence of reflections, which will produce fake edges. Pfeifer and Wiegers [6] proposed a capture system based on multiple light sources and multiple tool images acquisition. This

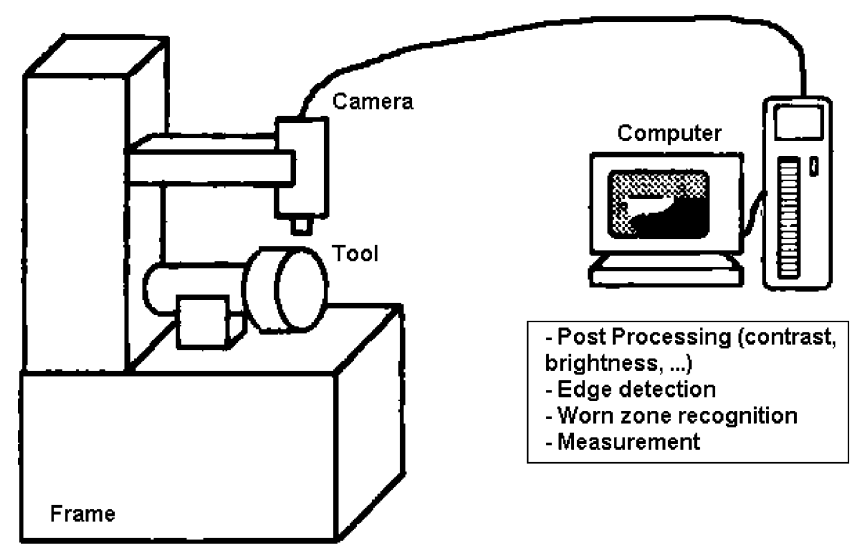

Fig. 1. Tool wear measurement system.

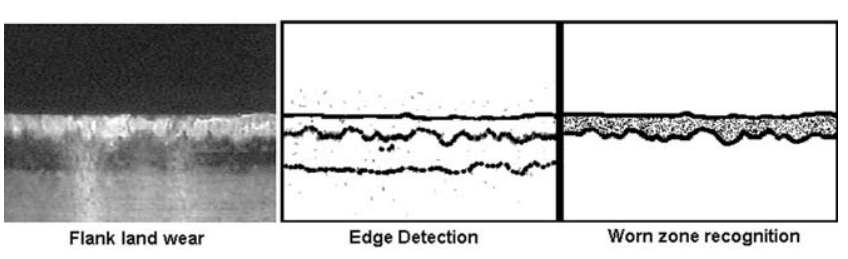

Fig. 2. Steps of worn-zone detection.

system is more robust to reflections and other disturbances since the worn zone is reconstructed using multiple images with different light sources. The camera settings (brightness, contrast, etc.) are important, but the image can be post-processed by the computer software. After post-processing the edges are enhanced by using a high-pass bi-dimensional filter. Several kinds of filter have been applied and tested for this purpose.

Several approaches to tool wear estimation have been found in literature. Tauno and Lembit [7] realized a portable monitoring system using a CCD camera and a portable computer. From a series of images taken from the tool, the software used a non-linear median filter to remove noise and a variation of Roberts filter operator for edge detection.

A neural network architecture for tool wear detection has been presented by Giardini et al. [8]. In this a digital camera collected images of the tool and the cutting zone has been used as an input for a perceptron. The perceptron is trained to detect whether the insert or the tool must be changed.

\section{WEARMON method for optical detection of tool wear}

Several edge detection algorithms have been tested for this application but the results have not been satisfactory for different reasons. First, the most known algorithms (Sobel, Roberts, etc.) are simple high-pass filters based on differences of small pixel sets. Thus, these algorithms tend to be very sensitive to 'light spots' and detect edges 

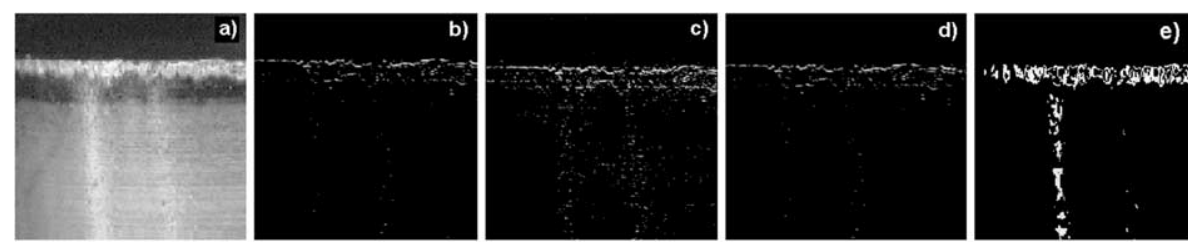

Fig. 3. Comparison of edge detection algorithms: (a) original image, (b) smoothed, (c) Sobel, (d) Laplace, (e) Previtt.

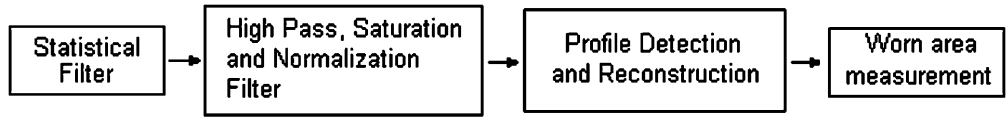

Fig. 4. The main steps of wear land detection algorithm.

wherever a spot is, while, for wear detection, pattern recognition algorithms seem to be more efficient. Moreover, the worn zone is very heterogeneous and a generic algorithm is not very efficient. As stated in Ref. [9], in noisy images it is necessary to use larger sets to do the comparison. An example of the results acquired by using these algorithms is illustrated in Fig. 3.

It can be seen that the edge detection algorithms Fig. 3(b)-(e) are not reliable solutions. For example, the Previtt algorithm Fig. 3(e) shows the nose of the tool (vertical) as tool wear. The best algorithm for edge detection is a combination of a low-pass and a high-pass filter [10]. In this research a new directional algorithm has been applied for tool worn area detection using a statistical filter. The algorithm is composed of several steps as shown in Fig. 4.

First, by using the statistical filter the edges of the image are detected. Given a reference pixel, the surrounding pixels are grouped in sets (for example, the pixel at the bottom and at the top of the reference pixel) and statistical parameters (mean $\mu$ and standard deviation $\sigma$ ) are calculated for each set and for each fundamental colour (red, green and blue). Then, set parameters are compared to parameters of the other set using statistical population comparison methods and $\Delta_{\text {edge }}$ for every fundamental colour is calculated, as shown in Eq. (1). All $\Delta_{\text {edge }}$ for the fundamental colours are then summed in order to compute $\Delta_{\text {edge }}$, Eq. (2). The higher the value of $\Delta_{\text {edge }}$ is, the greater is the probability that the pixel is an edge. The working principle is illustrated in Fig. 5

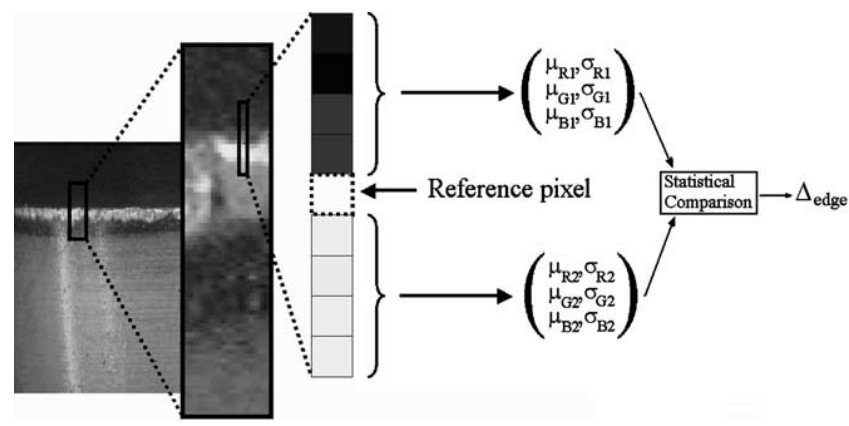

Fig. 5. The principle of statistic algorithm.

$$
\left\{\begin{array}{l}
\Delta_{\text {edge }}^{\mathrm{R}}=\frac{\mu_{\mathrm{R} 1}-\mu_{\mathrm{R} 2}}{\sqrt{\sigma_{\mathrm{R} 1}^{2}+\sigma_{\mathrm{R} 2}^{2}}} \\
\Delta_{\text {edge }}^{\mathrm{G}}=\frac{\mu_{\mathrm{G} 1}-\mu_{\mathrm{G} 2}}{\sqrt{\sigma_{\mathrm{G} 1}^{2}+\sigma_{\mathrm{G} 2}^{2}}} \\
\Delta_{\text {edge }}^{\mathrm{B}}=\frac{\mu_{\mathrm{B} 1}-\mu_{\mathrm{B} 2}}{\sqrt{\sigma_{\mathrm{B} 1}^{2}+\sigma_{\mathrm{B} 2}^{2}}} \\
\Delta_{\text {edge }}=\Delta_{\text {edge }}^{\mathrm{R}}+\Delta_{\text {edge }}^{\mathrm{G}}+\Delta_{\text {edge }}^{\mathrm{B}} .
\end{array}\right.
$$

In the second step, the $\Delta_{\text {edge }}$ values for every pixel are high pass filtered in order to reduce low values and normalize by using saturation. Then the profile of the wear land is detected by identifying the peaks of $\Delta_{\text {edge }}$ along rows and columns of the pixel matrix. After identification of the profile points, the profile is reconstructed by filtering scattered points and by interpolation. Lastly, the geometry characteristics of the wear land are calculated on the profile.

The main characteristic feature of this method is to be more sensible to pattern differences compared to other methods, which use smaller sets of pixel for comparison. This is very important due to the heterogeneity of the worn zone (see Fig. 6).

In Fig. 6 an example of the edges detected by applying the statistic algorithm to tool images is given. With this algorithm a good detection of the edges is possible. For example, in Fig. 6(b), it is possible to see three different profiles. The upper profile (1) is the cutting edge; the middle profile (2) is the interface between the worn zone (grey colour) and the oxidized zone (black colour); the
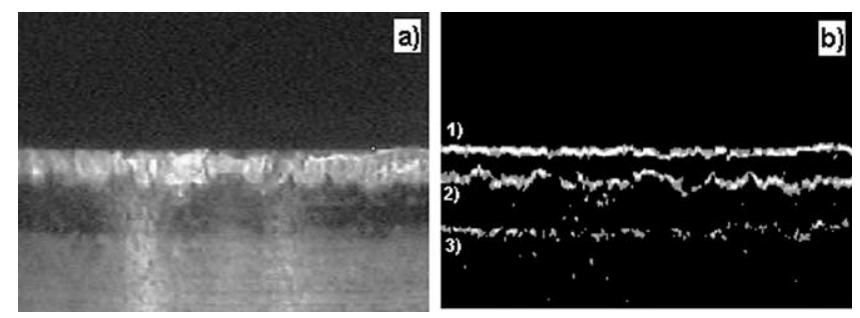

Fig. 6. Example of statistic algorithm results (a) acquired image, (b) detected edge. 
lower profile (3) is the interface between the oxidized zone and the tool surface (light grey colour).

After the edge detection, if the pictures are taken in the proper position, it is easy to measure the flank wear land in this case by measuring the thickness of the zone between the upper and the middle profile with some simple filtering and interpolation procedures.

\section{The WEARMON software}

In this work, an algorithm has been developed and implemented into the software for wear monitoring called WEARMON while the software has been installed on the 'SCOPEMAN' portable system. A portable computer and a camera with a 50× magnification lens compose the SCOPEMAN system. The system is capable of acquiring digital images sized $640 \times 480$, where every pixel has a dimension of about $10 \mu \mathrm{m}$. The images can be stored or processed immediately. The system is also capable of detecting the best algorithm functioning parameters and whether the detected worn area is close to reality or not.

The software user interface, Fig. 7, is very simple and allows the operator to take the measurement very easily and to see immediately how the algorithm has measured the worn zone.

\section{Method of validation}

The WEARMON software has been tested on over 150 different milling and turning worn tools and the obtained data have been compared to data obtained by optical microscopes. The comparison is shown in Fig. 8. It can be seen that the data obtained by applying the WEARMON software are very reliable.

Only $8 \%$ of cases of the system were not able to calculate the maximum flank wear land due to some disturbances on the cutting edge. In an attempt to be as close to reality as possible, only one picture was obtained for

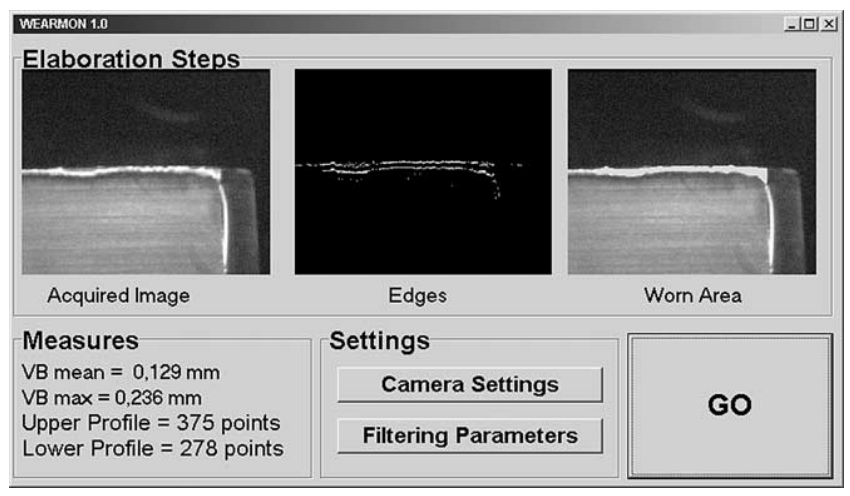

Fig. 7. The WEARMON software user interface.

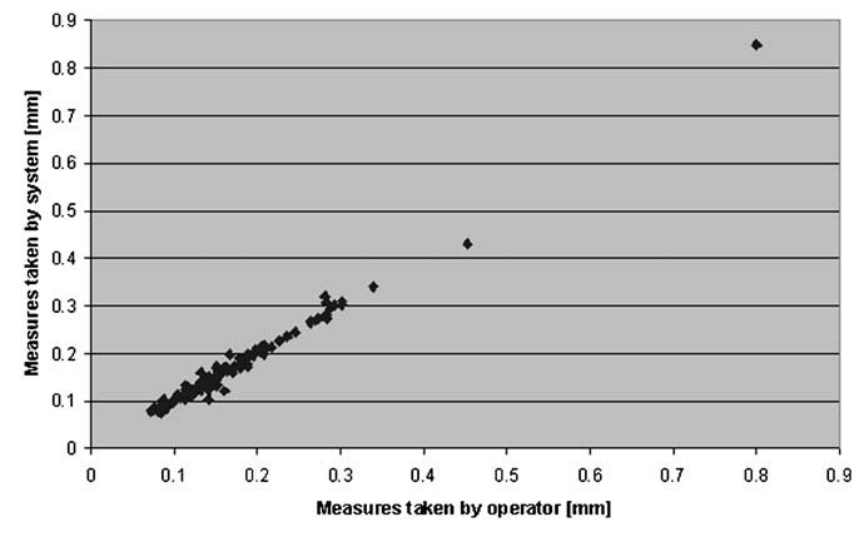

Fig. 8. Scatter of system measures against microscope measures.

every insert using the first acquired picture, considering the characteristics of the system in a future full-automated implementation without human feedback.

Because of the fixed pixel width $(10 \mu \mathrm{m})$, the accuracy decreases a great deal when the worn zone is very thin. Hence, the variance of the measured error is very high when the worn zone is thin, as can be seen in Fig. 9.

The operating range is between 0.1 and $2.5 \mathrm{~mm}$, which are the typical values of $\mathrm{VB}_{\max }$ for worn out tools. The accuracy of $10 \%$ for measurement error is enough to consider this system sufficiently reliable.

However, by changing the video source with a more sophisticated one, i.e. the pixel width less than $10 \mu \mathrm{m}$, there will be an increase in the accuracy of the measurement.

\section{Conclusions}

An innovative methodology and a system for wear measurement have been presented. This system is based on statistical filtering of images of the cutting edge. The statistical filter proved to be very efficient in comparison to standard filters for edges detection. The algorithm has

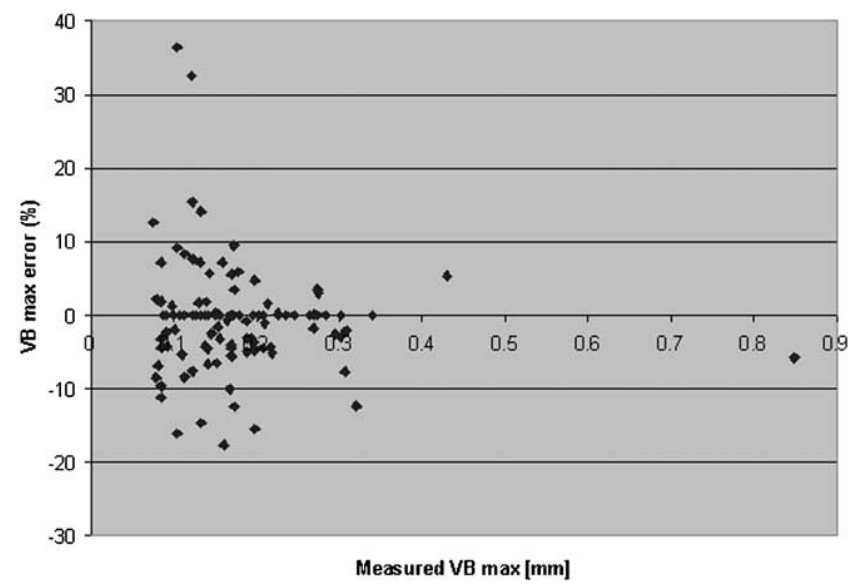

Fig. 9. Measure error against measure. 
been implemented in the WEARMON software, which can calculate the flank wear land, VB, from the wear patterns automatically. It was proved that results obtained with the system WEARMON are reliable except for small values of the tool wear. This problem can be overcome with an application of a more sophisticated video source. It would be of further interest to continue the investigation of the system by using more sophisticated video sources for industrial application.

\section{Acknowledgements}

The author would like to thank Professor E. Kuljanic and Dott. F. Miani for their contribution to this work. Special thanks to Dott. E. Guerra for his great help in the realization of the software.

\section{References}

[1] G. Byrne, D. Dornfeld, I. Inasaki, G. Ketteler, W. Koenig, R. Teti, Tool condition monitoring (TCM) - the status of research and industrial application, Keynote paper, Annals of the CIRP 44 (2) (1995) 541-567.

[2] E. Kuljanic, M. Sortino, Recent trend and developments in tool condition monitoring, advanced manufacturing systems and technology, in: CISM Courses and Lectures No. 437, Springer Wien, New York, 2002, pp. 15-36.

[3] G. Boothroyd, W.A. Knight, Fundamentals of Machining and Machine Tools, Dekker, New York, 1989.

[4] E. Dimla, Snr. Dimla. Sensor signals for tool-wear monitoring in metal cutting operations a review of methods, International Journal of Machine Tools and Manufacture 40 (2000) 1073-1098.

[5] V.C. Venkatesh, H. Chandrasekaran, Experimental Techniques in Metal Cutting, Prentice Hall, Englewood Cliffs, NJ, 1987.

[6] T. Pfeifer, L. Wiegers, Reliable tool wear monitoring by optimised image and illumination control in machine vision, Measurement 28 (2000) 209-218.

[7] O. Tauno, K. Lembit, Digital Tool Wear Measuring Video System, Estonia, 2000, http://mess.me.ttu.ee/Otto/DAAAM.pdf.

[8] C. Giardini, E. Ceretti, G. Maccarini, A neural network architecture for tool wear detection through digital camera observations, advanced manufacturing systems and technology, in: CISM Courses and Lectures No. 372, Springer Wien, New York, 1996, pp. 137-144.

[9] B. Jahne, Digital Image Processing, in: 2002, fifth ed., Springer, Berlin, 2002.

[10] W.K. Pratt, Digital Image Processing, Wiley, New York, 1978. 